\title{
Level-k Models Rationalize Overspending in Contests
}

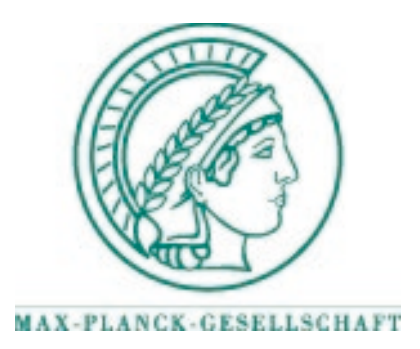

Max Planck Institute for

Tax Law and Public Finance

Department of Business and Tax Law Department of Public Economics

http://www.tax.mpg.de 
Working papers of the Max Planck Institute for Tax Law and Public Finance Research Paper Series serve to disseminate the research results of work in progress prior to publication to encourage the exchange of ideas and academic debate. Inclusion of a paper in the Research Paper Series does not constitute publication and should not limit publication in any other venue. The preprints published by the Max Planck Institute for Tax Law and Public Finance represent the views of the respective author(s) and not of the Institute as a whole. Copyright remains with the author(s).

Max Planck Institute for Tax Law and Public Finance

Marstallplatz 1

D-80539 Munich

Tel: $\quad+498924246-0$

Fax: $\quad+498924246-501$

E-mail:ssrn@tax.mpg.de

http://www.tax.mpg.de 


\title{
Level-k models rationalize overspending in contests*
}

\author{
Malin Arve $^{\dagger}$ and Marco Serena ${ }^{\ddagger}$
}

October 9, 2017

\begin{abstract}
The experimental evidence on contests often reports overspending of subjects as opposed to the Nash equilibrium. Explaining such phenomenon is a long-standing research question. We complement the alternative explanations proposed by the literature by providing a theoretical rationale for overspending which is based on the level-k model. This also bridges an open gap between the contest and auction literatures.
\end{abstract}

Keywords: Contest, Level-k reasoning

C72, D72

\section{Introduction}

The experimental literature on contests often reports significant overspending of subjects compared to the Nash equilibrium - see, Davis and Reilly (1998), Potters et al. (1998), Sheremeta and Zhang (2010), and Morgan et al. (2012), among others. We propose a theoretical rationale for overspending in Tullock contests (Tullock, 1980) which is based on the level-k model.

We adopt the basic version of a level-k model, which works as follows. A player of depth of reasoning $k$, in short a $L k$-player, believes that the other players are $L k-1$, and thus "best responds" to this belief. In particular, L1-players best respond to all other players playing the strategy of $L 0$-players, $L 2$-players best respond to all other players playing the strategy of $L 1$-players, and so on. ${ }^{1} L 0$-players are nonstrategic in

${ }^{*}$ We are grateful to Cédric Wasser and to the participants of the fifth informal research meeting at the Max Planck Institute for Tax Law and Public Finance for useful comments. Errors are ours.

$\dagger^{\dagger}$ HH Norwegian School of Economics. Email: malin.arve@nhh.no

${ }^{\ddagger}$ Max Planck Institute for Tax Law and Public Finance. Email: marco.serena@tax.mpg.de

${ }^{1}$ Some alternatives of behavior of types $L 2$ and higher have been proposed. For instance, in Stahl and Wilson (1994 and 1995) while $L 1$-players best respond to uniformly playing $L 0$-players, $L 2$-players best respond to a mixture of $L 0$-players and $L 1$-players, and so on. Another alternative is Ho et al. (1998), where $L k$-players best respond to an estimated mixture of players of lower $k$, via a one-parameter Poisson distribution. In the present paper, we choose the most simple and common approach that $L k$ best responds to all others been $L k-1$. 
that they form no beliefs over the opponents' actions and rather play an instinctive reaction to the game. It is common in the literature to assume that $L 0$-players uniformly mix over all possible actions ${ }^{2}$ and this is backed up by empirical findings. ${ }^{3}$

Furthermore, the literature on auctions often runs parallel to that on contests. Overbidding in auctions has also been documented empirically/experimentally (see, Kagel and Levin (2002) for an overview) and can in private-value auctions be rationalized by level-k models (Crawford and Iriberri, 2007). We bridge the existing gap between the auction and contest literature by showing that this can also be true in a contest environment.

In general, there are two main branches of theoretical models that have been proposed to explain overspending. ${ }^{4}$ The first branch of models drops the standard specification of the utility function of subjects - e.g., assuming an extra non-monetary utility derived either from winning (see Parco et al., 2005, for the case of contests and Cox et al. (1992) and Holt and Sherman (1994) for the case of auctions) or from relative payoff (e.g., Mago et al., 2014), or assuming that subjects assign a distorted value to the probability of winning (e.g., Baharad and Nitzan, 2008). The second branch of models, to which this paper belongs, suggests that players do not work out all the necessary steps to compute the Nash equilibrium — i.e., subjects are boundedly rational. There are two main approaches to bounded rationality models. Namely, quantal response equilibrium (QRE) models (see McKelvey and Palfrey, 1995), where the probability of a certain action is increasing in the expected payoff of that action and level-k models. Applications of QRE models to contests can be found in Sheremeta (2011), Chowdhury et al. (2014) and Lim et al. (2014). For auctions, overspending based on QRE is shown by Goeree et al. (2002). We again provide the missing link that shows that a similar result can be obtained in level-k contest models.

To our knowledge, the only paper applying level-k modeling to contests is Bernard (2010). He studies a two-player symmetric Tullock contest and shows that overspending does not occur. This creates a divide between the auction and contest literatures where results are not the same from one literature to the other. We ar-

\footnotetext{
${ }^{2}$ See, Stahl and Wilson (1994, 1995), Nagel (1993), Ho, Camerer, and Weigelt (1998), CostaGomes, Crawford, and Broseta (2001), Camerer et al. (2004), Costa-Gomes and Crawford (2006), Crawford and Iriberri (2007a) and Crawford et al. (2013; sections 2.4 and 5.1). An alternative approach is that $L 0$-players choose a salient or intuitive action, also known as a "focal point" (see Schelling, 1960).

${ }^{3}$ As reported by Crawford et al. (2013), "the evidence [...] generally supports level-k models in which players anchor beliefs in a uniform random $L 0 . "$

${ }^{4}$ Additionally, features of the experimental design could also explain overspending in contests (e.g., Chowdhury et al., 2014). For a comprehensive list of alternative explanations of the overspending observed in experimental contests see the recent survey by Dechenaux et al. (2015).
} 
gue that Bernard (2010) is a special case and extending the framework to either more players or allowing for asymmetric players yields an overspending result consistent with the aforementioned parallel between these two literatures.

Our main argument relies on the observation that in a two-player symmetric Tullock contest the Nash equilibrium is situated at the peak of the best reply function. In such a situation there is no scope for overspending since the Nash equilibrium is already at the maximum and there would be no assumption on the behavior of $L 0$-players that would make an $L k \geq 1$ player exert strictly more effort than in the Nash equilibrium, even if one would cherry-pick the action of $L 0$-players. ${ }^{5}$

However, it is sufficient to drop one of the two assumptions - namely, symmetry or two players - to find overspending already at the $L 1$ level of rationality, and the intuition relies on the Nash equilibrium no longer at the peak of the best reply function. In particular, we find that overspending occurs in two-players (sufficiently $^{6}$ ) asymmetric contests and in three-players symmetric contests; see, respectively, Proposition 2 and Proposition 4. The link between the contest and auction literature that we contribute to, is summarized in the following table.

Table 1. Does bounded rationality rationalize overspending...

\begin{tabular}{|l|l|l|}
\hline & QRE & Level-k \\
\hline ...in Auctions? & Yes: Goeree et al. (2002) & Yes: Crawford and Iriberri (2007) \\
\hline ...in Contests? & Yes: Sheremeta (2011), & No: Bernard (2010) \\
& Chowdhury et al. (2014), Lim et al. (2014) & Yes: present paper \\
\hline
\end{tabular}

Our theoretical results are also consistent with empirical evidence other than the possibility of overspending. First, the fact that overspending increases with the number of bidders (e.g., Sheremeta 2013) is consistent with our result that moving from two to three players makes overspending strictly positive. Second, the behavior's heterogeneity of contestants facing identical contests (e.g., Sheremeta and Zhang, 2010) is consistent with the fact that the depth of reasoning and the realization of the L0's random behavior may differ across players.

The structure of the paper is as follows. In Section 2 we introduce the standard model of Tullock contest. We then provide benchmark results for the Nash equilibrium as well as level-k results in the case of a two-player symmetric contest in

\footnotetext{
${ }^{5}$ As noted by Haile et al. (2008) among others, one could always find an arbitrarily cherrypicked behavior of $L 0$-players so as to rationalize any individually rational action of $L 1$-players, if no further restrictions are imposed. However, in the present paper, we tie our hands imposing that $L 0$-players randomize uniformly over the space of positive efforts with a given upper-bound. Thus, our exercise of rationalizing overspending for any given such upper-bound is non-trivial.

${ }^{6} \mathrm{~A}$ minimal level of asymmetry is required otherwise the Nash equilibrium would be too close to the peak of the best reply function.
} 
Section 3. In Section 4 we show that overspending may occur in two-player asymmetric contests and in Section 5 we prove that it occurs in three-player symmetric contests. Section 6 discusses the results and concludes. Proofs that do not follow directly from the main text are relegated to the Appendix.

\section{Model}

Consider a complete information Tullock-contest with $n$ risk-neutral players indexed by $i \in\{1, \ldots, n\}$. Players compete for a prize whose value we, without loss of generality, normalize to 1 . Each player $i$ chooses effort level $e_{i}$, and has a probability of winning the prize equal to

$$
p_{i}\left(e_{1}, \ldots, e_{n}\right)=\frac{e_{i}}{\sum_{j=1}^{n} e_{j}},
$$

with $p_{i}: \mathbb{R}_{+}^{n} \rightarrow[0,1], \forall i \in\{1, \ldots, n\} e_{i} \geq 0$. If all efforts are 0 the prize is awarded with a fixed probability strictly less than 1 to each player. However, this situation is never reached in equilibrium. The cost of effort is linear and the marginal cost equals $c_{i}>0$ for player $i$. Hence, player $i$ chooses $e_{i}$ to maximize

$$
\frac{e_{i}}{\sum_{j=1}^{n} e_{j}}-c_{i} e_{i}
$$

Throughout the paper, we denote by $e^{N E}$ the Nash equilibrium of such a game, and by $e^{L i}$ the effort of $L i$-players in the level-k version of such game. In level-k models, the most frequently observed types are typically $L 1$ and $L 2$, and higher levels are seldomly observed. ${ }^{7}$ Since our ultimate goal is to test whether at some $k$ overspending is rationalizable, and we find that at $k=1$ a player already overspends, we only characterize the level 0 and the level 1 here, and we briefly discuss the evolution of behavior of higher types in the final discussion.

While in games with compact action space the support over which $L 0$-players randomly chose their action is unambiguously defined, in contests the strategy space is the whole positive real line. Thus, we assume that $L 0$-players randomize uniformly over the interval $[0, \bar{e}]$ with $\bar{e}>0$. Notice that we take an agnostic view on $\bar{e}$, which we allow to be lower, but also greater, than the valuation of the prize. ${ }^{8}$

\footnotetext{
${ }^{7}$ For estimations of the distribution of subjects levels of reasoning see for instance Costa-Gomes, Crawford and Broseta (2001), Arad and Rubinstein (2012) and Kneeland (2015).

${ }^{8}$ In fact, some experimental evidence shows that subjects may bid more than the prize they
} 


\section{Benchmark: Nash equilibrium and two-player symmetric contests with level-k rationality}

Before presenting our results, we present the main benchmark, the Nash equilbrium with fully rational players, as well as a generalization of Bernard (2010)'s result for two-player symmetric contests with level-k rationality. These are the two outcomes that we contrast our results with.

Nash equilibrium under full rationality. With two heterogeneous players, the well-known unique Nash equilibrium of the full rationality model for player 1 is ${ }^{9}$

$$
\begin{aligned}
e_{1}^{N E} & =\frac{c_{2}}{\left(c_{1}+c_{2}\right)^{2}} \\
& =\frac{a}{(1+a)^{2} c_{1}}
\end{aligned}
$$

where we defined $a=c_{2} / c_{1}$, which is thus the asymmetry between contestants.

For player 2, the equilibrium level of effort is

$$
e_{2}^{N E}=\frac{1}{(1+a)^{2} c_{1}} .
$$

With $n$ homogeneous players with cost parameter $c_{i}=c, \forall i \in\{1, \ldots, n\}$, the unique Nash equilibrium of the full rationality model is

$$
e^{N E}=\frac{n-1}{n^{2} c}
$$

For $n=3$, this yields $e^{N E}=\frac{2}{9 c}$.

A full characterization of the equilibrium with $n$ players and asymmetry is, for instance, in Fullerton and McAfee (1999). Even with just three asymmetric players, there are parameter constellations for which not all contestants exert positive effort, and these endogenous participation issues would confound the results of our paper. In fact, our ultimate goal is to verify rationalizatility of overspending in Tullock contests, and the special cases of three symmetric players and two asymmetric players suffice for this goal.

Two-player symmetric contests with level-k rationality. We now consider the symmetric two-player contest with level-k rationality and prove the impos-

might win - e.g., Baye et al. (1999), Sheremeta and Zhang (2010), Mago and Sheremeta (2012), Morgan et al. (2012). $\bar{e}$ can be interpreted as the initial endowment of money that subjects are often given in experiments, and the fact that $L 0$-players choose randomly between 0 and their endowment is consistent with the empirical finding that overspending increases in the endowment (see the meta-study Sheremeta, 2013).

${ }^{9}$ See Konrad (2002) for a general presentation of Tullock contests under full rationality. 
sibility of overspending result. This benchmark thus generalizes the impossibility found in Bernard (2010) to any $(c, \bar{e})$, where $c$ is the marginal cost of effort for a player. ${ }^{10}$

An $L 1$-player believes that her rival is $L 0$ and thus plays uniformly over $[0, \bar{e}]$. Therefore, the FOC of $L 1$-players writes

$$
\begin{aligned}
\int_{0}^{\bar{e}} \frac{z}{\bar{e}\left(e^{B}+z\right)^{2}} d z & =c \\
& \Longleftrightarrow \frac{e^{B}}{e^{B}+\bar{e}}+\log \left[e^{B}+\bar{e}\right]-1-\log \left[e^{L 1}\right]=c \bar{e} \\
& \Longleftrightarrow \log \left[\frac{e^{B}+\bar{e}}{e^{B}}\right]-\frac{\bar{e}}{e^{B}+\bar{e}}=c \bar{e},
\end{aligned}
$$

where $e^{B}$ denotes the equilibrium strategy of L1 players in the generalized Bernard model.

The left-hand side (LHS) of (5) is strictly decreasing in $e^{B}$, tends to $\infty$ as $e^{B} \rightarrow 0$, and tends to 0 as $e^{B} \rightarrow \infty$. Thus, a unique interior equilibrium $e^{B}$ exists, and solves (5).

Since the LHS of (9) decreases in $e^{B}$,

$$
\begin{aligned}
e^{B} & >e^{N E} \\
& \Longleftrightarrow \log \left[\frac{e^{N E}+\bar{e}}{e^{N E}}\right]-\frac{\bar{e}}{e^{N E}+\bar{e}}>c \bar{e} \\
& \Longleftrightarrow \log [1+4 c \bar{e}]-\frac{4 c \bar{e}}{1+4 c \bar{e}}>c \bar{e} .
\end{aligned}
$$

The condition for overspending in (7) is equivalent to

$$
\log [1+4 c \bar{e}]-\frac{5 c \bar{e}+4(c \bar{e})^{2}}{1+4 c \bar{e}}>0
$$

Bernard (2010) assumes that $c=\bar{e}=1$, for which the above condition is violated, yielding his result of impossibility of overspending.

Routine algebra shows that the LHS of (8) decreases in $c \bar{e}$, and its value is 0 when $c \bar{e}=0$, thus $(8)$ never holds for any $(c, \bar{e})>0$. Hence, we have proven the following generalization of Bernard (2010)'s impossibility of overspending in symmetric twoplayer contest.

Proposition 1 Consider a symmetric Tullock contest with $n=2$. Then, $\nexists(c, \bar{e})$ such that the L1 level of effort is greater than the effort level at the fully rational

\footnotetext{
${ }^{10}$ Bernard (2010) studies the special case of $c=1$ and $\bar{e}=1$.
} 
Nash Equilibrium. That is, $e^{B} \leq e^{N E}$.

This impossibility result is also a direct consequence of the fact that in a twoplayer symmetric contest the fully rational Nash equilbrium is at the peak of the players' best response functions. Thus, overspending is impossible in such a setting, as already proven by Bernard (2010) in the special case of $c=\bar{e}=1$.

However, this result relies on two important assumptions, namely $n=2$ and $c_{1}=c_{2}=c$. In the next two sections we show that dropping either of these two assumptions yields overspending relative to the Nash equilibrium.

\section{Overspending in two-players asymmetric con- test}

The main point of this section is that introducing asymmetry in players' costs of effort rationalizes overspending. Since we do not make any assumption of the sort $c_{1}>c_{2}$ or $c_{1}<c_{2}$, we can without loss of generality focus on player 1 only. By symmetry we can get the same result for $c_{2}$. We thus consider that the $L 1$-players have marginal cost $c_{1}$.

A $L 1$-player believes that her rival is $L 0$ playing uniformly over $[0, \bar{e}]$. Therefore, it is straightforward to see that $c_{2}$ does not enter into the condition of optimal $e^{L 1}$ and the FOC of $L 1$-players writes

$$
\begin{aligned}
& \int_{0}^{\bar{e}} \frac{z}{\bar{e}\left(e^{L 1}+z\right)^{2}} d z=c_{1} \\
\Longleftrightarrow & \frac{e^{L 1}}{e^{L 1}+\bar{e}}+\log \left[e^{L 1}+\bar{e}\right]-1-\log \left[e^{L 1}\right]=c_{1} \bar{e} \\
\Longleftrightarrow & \log \left[\frac{e^{L 1}+\bar{e}}{e^{L 1}}\right]-\frac{\bar{e}}{e^{L 1}+\bar{e}}=c_{1} \bar{e} .
\end{aligned}
$$

As in the benchmark case with symmetric costs, we can show that the LHS of (9) strictly decreases in $e^{L 1}$, tends to $\infty$ as $e^{L 1} \rightarrow 0$, and tends to 0 as $e^{L 1} \rightarrow \infty$. Thus, a unique interior equilibrium $e^{L 1}$ exists, and solves (9).

Since the LHS of (9) decreases in $e^{L 1}$,

$$
\begin{aligned}
e^{L 1} & >e^{N E} \\
& \Longleftrightarrow \log \left[\frac{e^{N E}+\bar{e}}{e^{N E}}\right]-\frac{\bar{e}}{e^{N E}+\bar{e}}>c_{1} \bar{e} \\
& \Longleftrightarrow \log \left[1+\frac{(a+1)^{2} c_{1} \bar{e}}{a}\right]-\frac{(a+1)^{2} c_{1} \bar{e}}{a+(a+1)^{2} c_{1} \bar{e}}>c_{1} \bar{e}
\end{aligned}
$$




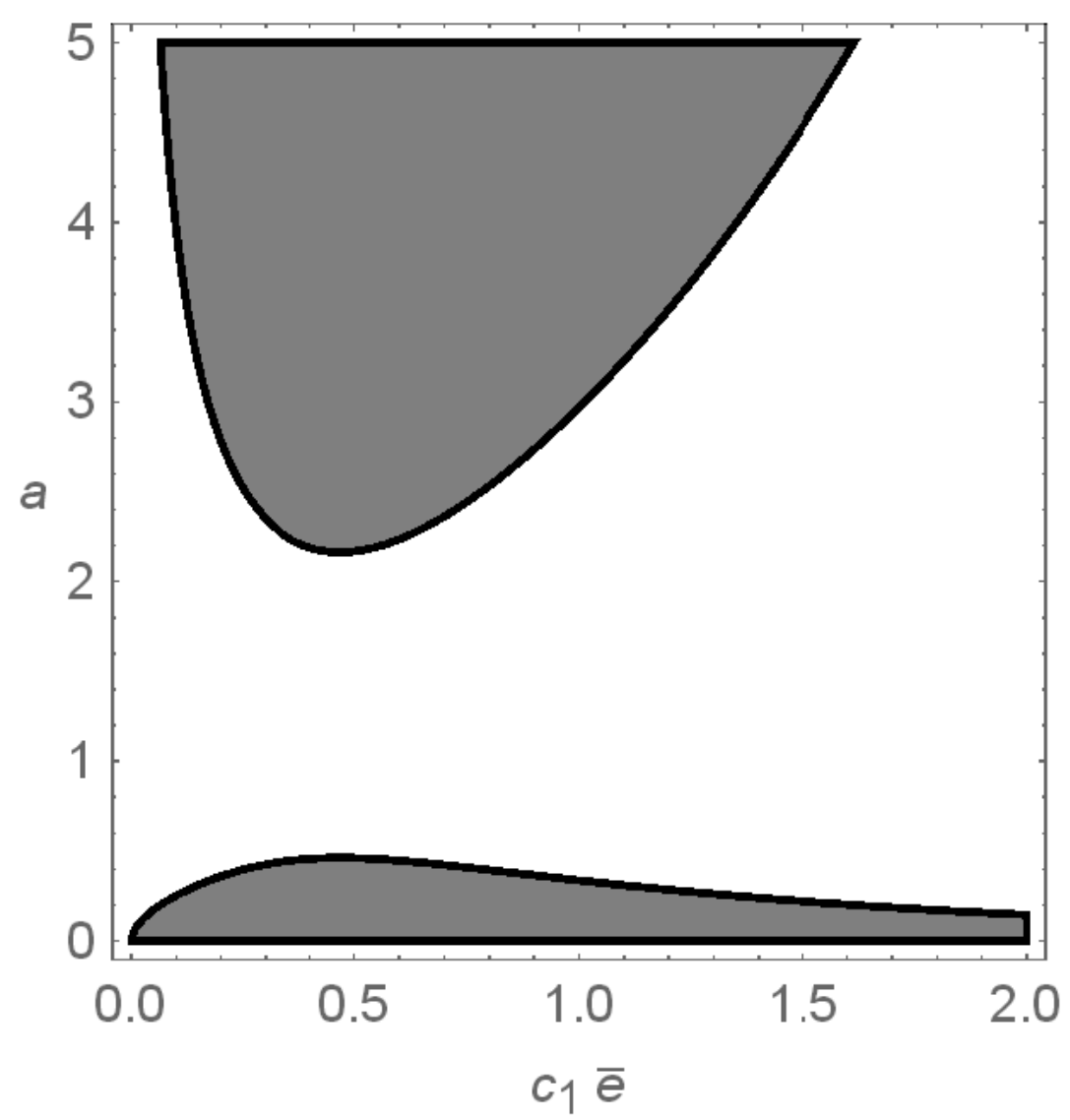

Figure 1: Region in the $\left(c_{1} \bar{e}, a\right)$-space where in a two-player contest $e^{L 1}>e^{N E}$, that is, the L1 level of effort is greater than the Nash equilibrium level.

where in the last step we used (3). Condition (11) can be plotted in two dimensions, namely $\left(c_{1} \bar{e}, a\right)$ and this is shown in Figure 1 . The shaded area represents combinations $\left(c_{1} \bar{e}, a\right)$ such that the L1 level of effort is greater than the Nash equilibrium level.

If $a \rightarrow \infty$, the LHS of (11) tends to $\infty$, and thus for any finite value of $c_{1} \bar{e}, \exists a$ sufficiently large so that (11) holds, and overspending occurs. Proving the opposite, namely that for any finite value of $a \geq \bar{a}, \exists c_{1} \bar{e}$ that makes (11) hold, is more tricky and we prove it in Lemma 5 in the Appendix, which also characterizes $\bar{a}$ (i.e., $\bar{a} \approx 2.16258$ ). Because $a$ measures the relative difference between $c_{1}$ and $c_{2}$, by symmetry we also get a lower bound $\underline{a}$ below which this is true. Thus, we have proven the following.

Proposition 2 Consider a Tullock contest with $n=2$ with parameters $\left(c_{1}, c_{2}, \bar{e}\right)$. For any two values of these parameters (if $c_{1}$ and $c_{2}$ are the chosen paramenters, it also has to be that $c_{2} / c_{1} \notin(\underline{a}, \bar{a})$ with $\underline{a} \approx 0.4624$ and $\left.\bar{a} \approx 2.1626\right)$, there exists $a$ value of the remaining parameter such that the L1 level of effort is greater than the 
Nash equilibrium level, i.e. $e^{L 1}>e^{N E}$.

Note that a minimum level of asymmetry between players is needed to rationalize overspending because otherwise the Nash equilibrium would be too close to the peak of the best reply function. Overspending in the asymmetric level-k Tullock contest can happen both by a weak or strong player who has sufficiently low or high cost compared to its competitor.

\section{Overspending in three-players symmetric con- test}

The main point of this section is that introducing a third player in a symmetric contest also rationalizes overspending. An L1-player believes that her two rivals are $L 0$-players playing uniformly over $[0, \bar{e}]$. A Tullock contest is an aggregative game ${ }^{11}$ in that (2) depends only on $e_{1}$ and on the sum of the rival's efforts $e_{2}+e_{3}$. For this reason, L1-players who are up against two rivals behaves as if up against one rival whose effort follows a triangular distribution $Z$ in $[0,2 \bar{e}]$, that is, the distribution of the sum of the two uniform random variables both in $[0, \bar{e}]$, namely:

$$
f_{Z}(z)=\left\{\begin{array}{c}
\frac{z}{\overline{\bar{e}}^{2}} \text { if } z \in[0, \bar{e}], \\
\frac{2 \bar{e}-z}{\bar{e}^{2}} \text { if } z \in[\bar{e}, 2 \bar{e}] .
\end{array}\right.
$$

Therefore, the FOC of the $L 1$-players writes

$$
\int_{0}^{\bar{e}} \frac{z^{2}}{\bar{e}^{2}\left(e^{L 1}+z\right)^{2}} d z+\int_{\bar{e}}^{2 \bar{e}} \frac{(2 \bar{e}-z) z}{\bar{e}^{2}\left(e^{L 1}+z\right)^{2}} d z=c .
$$

The following Lemma gives an alternative, but equivalent statement of this condition.

Lemma 3 (12) is equivalent to

$$
e^{L 1} \log \left[e^{L 1}\right]-\left(\bar{e}+2 e^{L 1}\right) \log \left[\bar{e}+e^{L 1}\right]+\left(\bar{e}+e^{L 1}\right) \log \left[2 \bar{e}+e^{L 1}\right]=\frac{\bar{e}^{2} c}{2} .
$$

Notice first, that the LHS of (13) decreases in $e^{L 1}$. The details of this proof is found in Lemma 6 in the Appendix. Second, as $e^{L 1} \rightarrow 0$, the LHS of (13) approaches $\bar{e} \log 2>0$, and as $e^{L 1} \rightarrow \infty$, it approaches 0 . Thus, there exists a unique equilibrium. Interiority is guaranteed by $2 \log 2>\bar{e} c$.

\footnotetext{
${ }^{11}$ Aggregative games are pioneered by Selten (1970).
} 
Since the LHS of (13) decreases in $e^{L 1}, e^{L 1}>e^{N E}$ if and only if the LHS of (13) with $e^{N E}$ instead of $e^{L 1}$ is greater than the RHS.

We next show that $\forall \bar{e}>0$, there exists a $c>0$ such that $e^{L 1}>e^{N E}$, and vice versa, $\forall c>0$ there is an $\bar{e}>0$ such that $e^{L 1}>e^{N E}$. An easy way to do so is to impose the following simplifying assumption

$$
\bar{e} c=\frac{1}{4}
$$

in condition (13) above since for any $\bar{e}$ or $c$ this pins down the value of the other parameter. Notice that (14) satisfies the interiority condition $2 \log 2>\bar{e} c$ found above. The intuition behind condition (14) is that in order to rationalize overspending one has to trade-off $\bar{e}$ and $c$. In fact, high expected effort from $L 0$-players (i.e., high $\bar{e}$ ) discourages L1-players, and thus has to be compensated by low marginal cost of effort $c$ to guarantee that she still exerts high effort. Similarly, costly effort (i.e., high $c$ ) discourages $L 1$-players, but this is compensated when $L 0$-players exert sufficiently low effort (i.e., low $\bar{e}$ ).

Thus, under condition (14), using (4) we obtain $e^{N E}=\frac{8 \bar{e}}{9}$, and thus:

$$
\begin{aligned}
e^{L 1} & >e^{N E} \\
& \Longleftrightarrow \frac{8 \bar{e}}{9} \log \left[\frac{8 \bar{e}}{9}\right]-\left(\bar{e}+\frac{16 \bar{e}}{9}\right) \log \left[\bar{e}+\frac{8 \bar{e}}{9}\right]+\left(\bar{e}+\frac{8 \bar{e}}{9}\right) \log \left[2 \bar{e}+\frac{8 \bar{e}}{9}\right]>\frac{\bar{e}}{8} \\
& \Longleftrightarrow \frac{8}{9} \log \left[\frac{8 \bar{e}}{9}\right]-\frac{25}{9} \log \left[\frac{17 \bar{e}}{9}\right]+\frac{17}{9} \log \left[\frac{26 \bar{e}}{9}\right]>\frac{1}{8} \\
& \Longleftrightarrow \frac{8}{9} \log \left[\frac{8 \bar{e}}{9}\right]-\frac{8}{9} \log \left[\frac{17 \bar{e}}{9}\right]-\frac{17}{9} \log \left[\frac{17 \bar{e}}{9}\right]+\frac{17}{9} \log \left[\frac{26 \bar{e}}{9}\right]>\frac{1}{8} \\
& \Longleftrightarrow \frac{8}{9} \log \left[\frac{8}{17}\right]+\frac{17}{9} \log \left[\frac{26}{17}\right]>\frac{1}{8} .
\end{aligned}
$$

whose LHS equals approximately 0.133 , and thus we have proven the following.

Proposition 4 Consider a symmetric Tullock contest with $n=3$ under a certain pair of parameters $(c, \bar{e})$. Arbitrarily pick one out of these two parameters. Then, there exists a value of the remaining parameter such that the L1 level of effort is greater than the Nash Equilibrium level, i.e. $e^{L 1}>e^{N E}$.

Note that having three players as opposed to two players shifts the NE sufficiently far from the peak of the best reply function, and this yields per se sufficient asymmetry to rationalize overspending. In other words, we do not need further assumptions on sufficient asymmetry, like $a \geq \bar{a}$ or $a \leq \underline{\mathrm{a}}$ in Proposition 2 .

In Figure 2 we plot in the $(c, \bar{e})$-space the region where $e^{L 1}>e^{N E}$. The dashed line is condition (14), which we used to prove Proposition 4. 


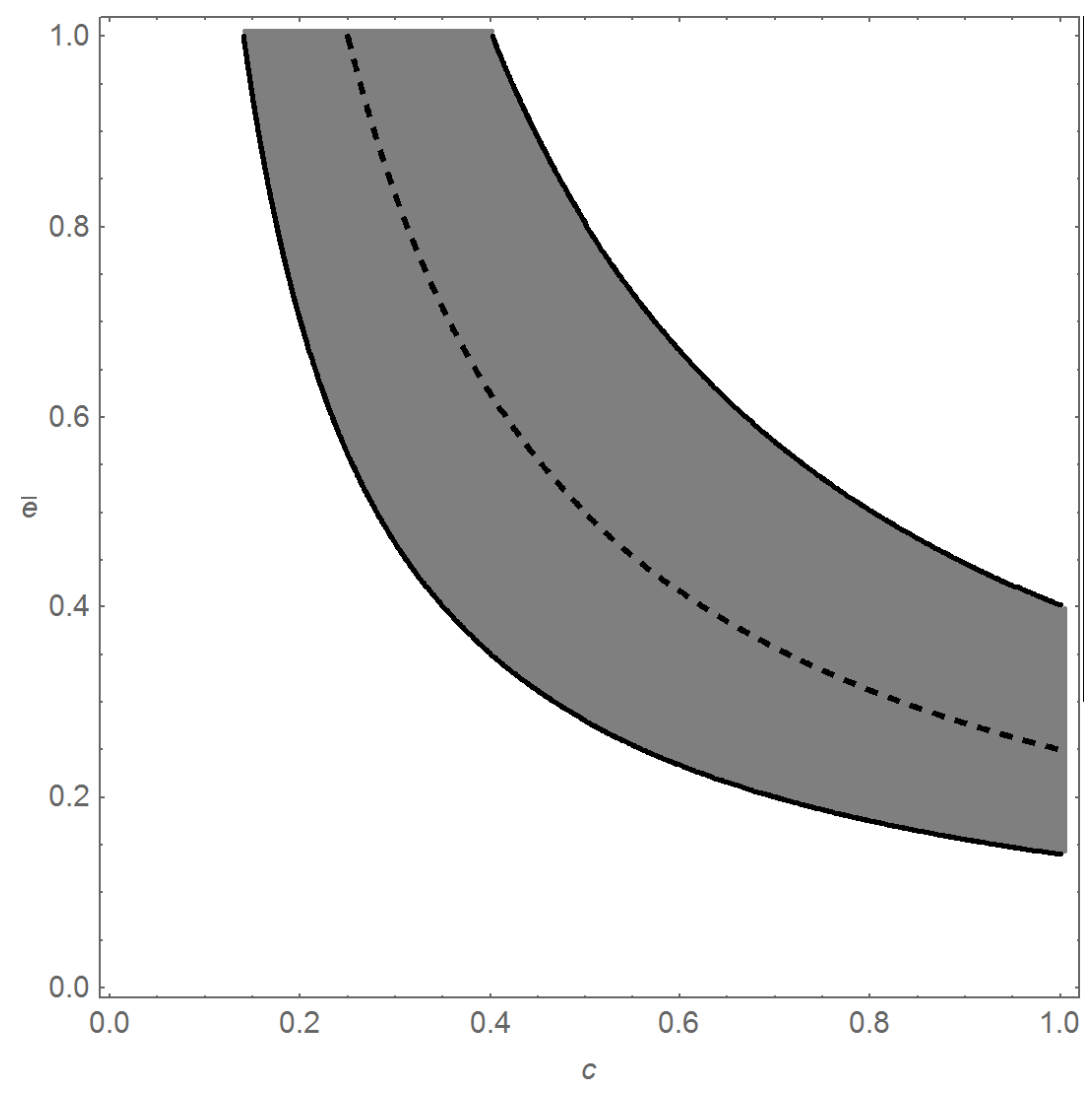

Figure 2: Region in the $(c, \bar{e})$-space where in a three-player contest $e^{L 1}>e^{N E}$, that is, the L1 level of effort is greater than the Nash equilibrium level. The dashed line is condition $\bar{e} c=\frac{1}{4}$.

\section{Discussion}

This paper shows that the result of overspending compared to the Nash equilibrium found both in experiments and in the auction literature, can also be rationalized by level-k models. We thereby bridge a gap between the auction and contest literature, two literatures that usually work in parallel.

The main analysis carried out in the present paper focused on the $L 1$ level of effort since this turned out to be sufficient to rationalize overspending. However, one might wonder about the level of efforts of $L k$-players with $k>1$. In particular, since we know from empirical evidence that the most frequently observed types are typically $L 1$ and $L 2$ (see footnote 7 ), it is important to focus also on $k=2$.

The effort of an $L 2$ is given by the iteration of a strictly concave best reply function of the form $e^{L 2}=F\left(e^{L 1}\right)$, with a unique maximum, say $e^{\max }$. More precisely, 


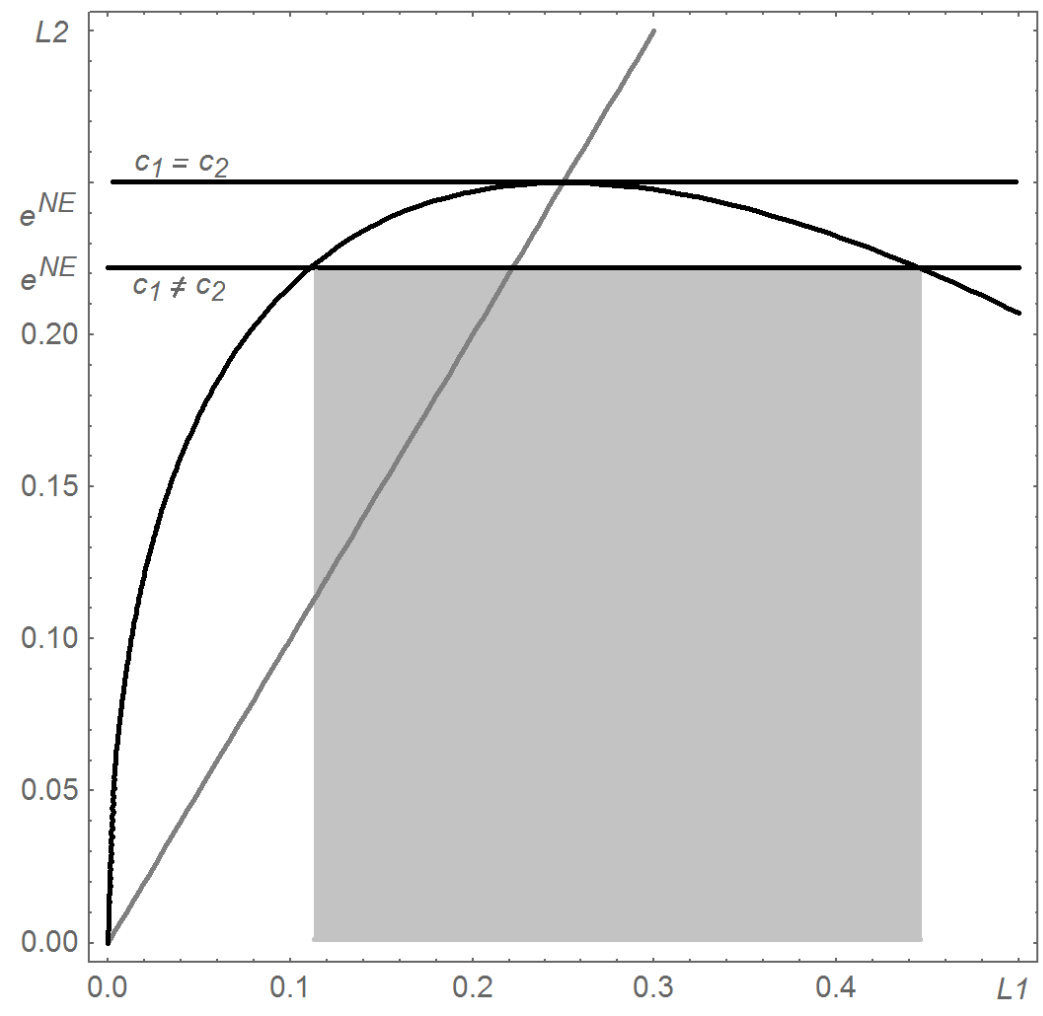

Figure 3: Efforts of $L 1$ and $L 2$ are on the axis. The horizontal line represents the $\mathrm{NE}$ in the three cases $c_{1}>c_{2}, c_{1}=c_{2}, c_{1}<c_{2}$. The gray region represents the $L 1$ effort such that, in case of $c_{1} \neq c_{2}, L 2$ overspends.

the best reply function of $L k$-players to a certain effort of $L k-1$-players is

$$
e_{1}^{k}=-(n-1) e_{2}^{k-1}+\sqrt{\frac{(n-1) e_{2}^{k-1}}{c_{1}}}
$$

and it reaches its maximum when $e_{1}^{k-1}=\frac{1}{4 c_{1}(n-1)} \equiv e_{1}^{\max }$.

If there are two asymmetric players, $e_{1}^{N E} \leq e^{\max }{ }^{12}$ If $c_{1} \neq c_{2}$ there is an intermediate region for $e^{L 1}$ such that overspending occurs at $L 2$ (see Figure 3 below), and at the same time even the $L 1$ may be overspending.

If there are three symmetric players, $e^{N E}>e^{\max },{ }^{13}$ and thus $L 2$ does not overspend.

Therefore we find that, while an $L 1$ may overspend in both cases of two asymmetric contestants and three symmetric contestants, only in the former case an $L 2$ may overspend. In fact, it is intuitive that it is harder to rationalize overspending for more sophisticated (L2) players as more sophisticated players play closer to the

\footnotetext{
${ }^{12}$ From (3), $e_{1}^{N E}=\frac{c_{2}}{\left(c_{1}+c_{2}\right)^{2}}$ and from the previous footnote $e^{\max }=\frac{1}{4 c_{1}}$, thus $e^{N E} \leq e^{\max }$.

${ }^{13}$ From (4), $e^{N E}=\frac{1}{8 c}$ and since $e^{\max }=\frac{1}{8 c}$, thus $e^{N E}>e^{\max }$.
} 
Nash equilibrium. This result thus provides a tight link between sophistication and overspending.

\section{A Appendix}

Lemma $5 \forall a \geq \bar{a}$ and $\forall a \leq \underline{a}, \exists c_{1} \bar{e}$ such that (11) holds. In particular, $\bar{a} \approx 2.1626$ and $\underline{a}=0.4624$.

Proof of Lemma 5. Consider Figure 1. We characterize the unique minimum $\bar{a}$ of the function in the LHS of (11). The minimum is where

$$
\begin{aligned}
& \frac{\partial}{\partial c_{1} \bar{e}}\left[\log \left[1+\frac{(a+1)^{2} c_{1} \bar{e}}{a}\right]-\frac{(a+1)^{2} c_{1} \bar{e}}{a+(a+1)^{2} c_{1} \bar{e}}-c_{1} \bar{e}\right]=0 \\
& \Longleftrightarrow \frac{a(a+1)^{2}}{a+(a+1)^{2} c_{1} \bar{e}}-\frac{(a+1)^{2}}{\left[a+(a+1)^{2} c_{1} \bar{e}\right]^{2}}-1=0 \\
& \Longleftrightarrow\left[a+(a+1)^{2} c_{1} \bar{e}\right]^{2}-a(a+1)^{2}\left[a+(a+1)^{2} c_{1} \bar{e}\right]+(a+1)^{2}=0
\end{aligned}
$$

And the solution of the above together with (11) characterizes the unique minimum. Namely, $\left(c_{1} e, \bar{a}\right) \approx(0.467586,2.1626)$. Swapping players' indexes, one can find the maximum of the bottom shaded area of Figure $1, \underline{a}=1 / \bar{a}=0.4624$.

\section{Proof of Lemma 3.}

$$
\begin{gathered}
\int_{0}^{\bar{e}} \frac{z^{2}}{\bar{e}^{2}\left(e^{L 1}+z\right)^{2}} d z+\int_{\bar{e}}^{2 \bar{e}} \frac{(2 \bar{e}-z) z}{\bar{e}^{2}\left(e^{L 1}+z\right)^{2}} d z=c \\
\Longleftrightarrow \quad \int_{0}^{\bar{e}}\left[1-\frac{2 e^{L 1} z+\left(e^{L 1}\right)^{2}}{\left(e^{L 1}+z\right)^{2}}\right] d z-\int_{\bar{e}}^{2 \bar{e}}\left[1-\frac{2 e^{L 1} z+\left(e^{L 1}\right)^{2}}{\left(e^{L 1}+z\right)^{2}}\right] d z+\int_{\bar{e}}^{2 \bar{e}} \frac{2 \bar{e} z}{\left(e^{L 1}+z\right)^{2}} d z=\bar{e}^{2} c \\
\Longleftrightarrow \quad-\bar{e}-e^{L 1}\left[\frac{e^{L 1}}{e^{L 1}+z}+2 \log \left(e^{L 1}+z\right)\right]_{0}^{\bar{e}}-\bar{e}+e^{L 1}\left[\frac{e^{L 1}}{e^{L 1}+z}+2 \log \left(e^{L 1}+z\right)\right]_{\bar{e}}^{2 \bar{e}}+ \\
\quad-e^{L 1}\left[\frac{-\bar{e}}{e^{L 1}+\bar{e}}+2 \log \frac{e^{L 1}+\bar{e}}{e^{L 1}}\right]+e^{L 1}\left[\frac{e^{L 1}}{e^{L 1}+2 \bar{e}}-\frac{e^{L 1}}{e^{L 1}+\bar{e}}+2 \log \frac{e^{L 1}+2 \bar{e}}{e^{L 1}+\bar{e}}\right]_{\bar{e}}=\bar{e}^{2} c \\
+2 \bar{e}\left[\frac{e^{L 1}}{e^{L 1}+2 \bar{e}}-\frac{e^{L 1}}{e^{L 1}+\bar{e}}+\log \frac{e^{L 1}+2 \bar{e}}{e^{L 1}+\bar{e}}\right]=\bar{e}^{2} c
\end{gathered}
$$




$$
\begin{array}{r}
\Longleftrightarrow \quad e^{L 1}\left[\frac{e^{L 1}}{e^{L 1}+2 \bar{e}}-\frac{e^{L 1}-\bar{e}}{e^{L 1}+\bar{e}}+2 \log \frac{e^{L 1}+2 \bar{e}}{e^{L 1}+\bar{e}}-2 \log \frac{e^{L 1}+\bar{e}}{e^{L 1}}\right]+ \\
+2 \bar{e}\left[\frac{e^{L 1}}{e^{L 1}+2 \bar{e}}-\frac{e^{L 1}}{e^{L 1}+\bar{e}}+\log \frac{e^{L 1}+2 \bar{e}}{e^{L 1}+\bar{e}}\right]=\bar{e}^{2} c \\
\Longleftrightarrow \quad e^{L 1}\left[\frac{2 \bar{e}^{2}}{\left(e^{L 1}+2 \bar{e}\right)\left(e^{L 1}+\bar{e}\right)}+2 \log \frac{e^{L 1}+2 \bar{e}}{e^{L 1}+\bar{e}}-2 \log \frac{e^{L 1}+\bar{e}}{e^{L 1}}\right]+ \\
+2 \bar{e}\left[-\frac{e^{L 1} \bar{e}}{\left(e^{L 1}+2 \bar{e}\right)\left(e^{L 1}+\bar{e}\right)}+\log \frac{e^{L 1}+2 \bar{e}}{e^{L 1}+\bar{e}}\right]=\bar{e}^{2} c \\
e^{L 1}\left[\log \frac{e^{L 1}+2 \bar{e}}{e^{L 1}+\bar{e}}-\log \frac{e^{L 1}+\bar{e}}{e^{L 1}}\right]+\bar{e}\left[\log \frac{e^{L 1}+2 \bar{e}}{e^{L 1}+\bar{e}}\right]
\end{array}
$$

Lemma 6 The LHS of (13) decreases in $e^{L 1}$.

\section{Proof of Lemma 6.}

$$
\begin{aligned}
& \frac{\partial}{\partial e^{L 1}}\left(e^{L 1} \log \left[e^{L 1}\right]-\left(a+2 e^{L 1}\right) \log \left[a+e^{L 1}\right]+\left(a+e^{L 1}\right) \log \left[2 a+e^{L 1}\right]\right)<0 \\
\Longleftrightarrow & \log \left[e^{L 1}\right]+1-2 \log \left[a+e^{L 1}\right]-\frac{a+2 e^{L 1}}{a+e^{L 1}}+\log \left[2 a+e^{L 1}\right]+\frac{a+e^{L 1}}{2 a+e^{L 1}}<0 \\
\Longleftrightarrow & \log \left[e^{L 1}\right]+1-2 \log \left[a+e^{L 1}\right]-1+\frac{e^{L 1}}{a+e^{L 1}}+\log \left[2 a+e^{L 1}\right]+1-\frac{a}{2 a+e^{L 1}}<0 \\
\Longleftrightarrow & 1+\frac{e^{L 1}}{a+e^{L 1}}-\frac{a}{2 a+e^{L 1}}+\log \left[\left(\frac{e^{L 1}}{a+e^{L 1}}\right)\left(\frac{2 a+e^{L 1}}{a+e^{L 1}}\right)\right]<0 \\
\Longleftrightarrow & \frac{a^{2}}{\left(a+e^{L 1}\right)\left(2 a+e^{L 1}\right)}+\log \left[\left(1-\frac{\bar{e}}{a+e^{L 1}}\right)\left(1+\frac{a}{a+e^{L 1}}\right)\right]<0 \\
\Longleftrightarrow & \frac{a^{2}}{\left(a+e^{L 1}\right)\left(2 a+e^{L 1}\right)}+\log \left[1-\left(\frac{a}{a+e^{L 1}}\right)^{2}\right]<0
\end{aligned}
$$

and the standard logarithm inequality $\log (1-x) \leq-x$ under $x \in[0,1)$ implies

$$
\frac{a^{2}}{\left(a+e^{L 1}\right)\left(2 a+e^{L 1}\right)}-\left(\frac{a}{a+e^{L 1}}\right)^{2}<0
$$

which trivially holds.

\section{References}

[1] Arad, A. and Rubinstein, A. (2012) "The 11-20 Money Request Game: A Level-k Reasoning Study", American Economic Review, 102, 3561-3573. 
[2] Baharad, E. and S. Nitzan (2008) "Contest Efforts in Light of Behavioural Considerations," The Economic Journal, 118, 2047-2059.

[3] Baye, M., D. Kovenock, and C. de Vries, (1999) "The Incidence of Overdissipation in Rent-Seeking Contests," Public Choice, 99, pp. 439-454.

[4] Mark, B. (2010) "Level-k reasoning in contests", Economic Letters, 149-152.

[5] Bosch-Domènech, A., J. M. Montalvo, R. Nagel and A. Satorra (2002) "One, Two, (Three), Infinity, ...: Newspaper and Lab Beauty-Contest Experiments", American Economic Review, 92(5): 1687-1701.

[6] Camerer C. T., T. H. Ho and J. K. Chong (2004): "A Cognitive Hierarchy Model of Games," Quarterly Journal of Economics, 119, 861-898.

[7] Chowdhury, S. M., Sheremeta, R. M., and Turocy, T. L. (2014). "Overbidding and overspreading in rentseeking experiments: Cost structure and prize allocation rules" Games and Economic Behavior., 87, 224-238.

[8] Costa-Gomes, M., V. Crawford and B. Broseta (2001) "Cognition and Behavior in Normal-Form Games: An Experimental Study", Econometrica, vol. 69, 2001, p. 1193-1235.

[9] Cox, J., Smith, V. L., and J. M. Walker (1992). "Theory and misbehavior of first-price auctions: comment", The american economic review, 82 (5), 13921412 .

[10] Crawford, V., and N. Iriberri (2007) "Level-k Auctions: Can a Nonequilibrium Model of Strategic Thinking Explain the Winner's Curse and Overbidding in Private-Value Auctions?" Econometrica 75 (6): 1721-70.

[11] Crawford, V., M. Costa-Gomes and N. Iriberri (2013) "Structural Models of Nonequilibrium Strategic Thinking: Theory, Evidence, and Applications." Journal of Economic Literature, 51(1):5-62.

[12] Davis, D, and Reilly, R. (1998) "Do many cooks always spoil the stew? An experimental analysis of rent seeking and the role of a strategic buyer". Public Choice, 95, 89-115.

[13] Dechenaux, E., D. Kovenock and R. Sheremeta (2015) "A survey of experimental research on contests, all-pay auctions and tournaments". Experimental Economics, Volume 18, Issue 4, pp 609-669. 
[14] Frederick, S. (2005). "Cognitive reflection and decision making". Journal of Economic Perspectives, 19, 25-42.

[15] Fullerton, R. L., and R. P. McAfee (1999). "Auctionin Entry into Tournaments," Journal of Political Economy, Vol. 107, No. 3, 573-605.

[16] Goeree, J. K., C. A. Holt and T. R. Palfrey (2002). "Quantal Response Equilibrium and Overbidding in Private-Value Auctions", Journal of Economic Theory, Volume 104, Issue 1, p. 247-272.

[17] Haile, P. A., A. Hortaçsu and G. Kosenok (2008) "On the Empirical Content of Quantal Response Equilibrium." American Economic Review, 98(1): 180-200.

[18] Ho, T. H., C. Camerer and K. Weigelt (1998) "Iterated Dominance and Iterated Best-response in p-Beauty Contests," American Economic Review, 88, 947-969.

[19] Holt, C.A., and R. Sherman (1994) "The Loser's Curse," American Economic Review, 84,642-652.

[20] Kagel, J., Levin, D., 2002. Bidding in common-value auctions: A survey of experimental research. Common value auctions and the winner's curse 1, 184 .

[21] Kahneman, D. (2011). "Thinking, fast and slow". New York, NY: Farrar, Straus and Giroux.

[22] Kneeland, T. (2015) "Identifying Higher-order Rationality" Econometrica, Vol. $83,2065-2079$

[23] Konrad, K. (2002) "Investment in the absence of property rights; the role of incumbency advantages". European Economic Review, 46: 1521-1537.

[24] Lim, W., Matros, A., and Turocy, T. L. (2014) "Bounded rationality and group size in Tullock contests: Experimental evidence". Journal of Economic Behavior \& Organization, 99, 155-167.

[25] Mago, S. D., and Sheremeta, R. M. (2012) "Multi-Battle Contests: An Experimental Study", SSRN Working Paper.

[26] Mago, S. D., Savikhin, A. C., and Sheremeta, R. M. (2014). "Facing your opponents: Social identification and information feedback in contests." Journal of Conflict Resolution.

[27] McKelvey, R., and Palfrey, T. (1995). "Quantal response equilibria for normal form games". Games and Economic Behavior, 10, 6-38. 
[28] Morgan, J., Orzen, H., and Sefton, M. (2012). "Endogenous entry in contests". Economic Theory, 51, 435-463.

[29] Nagel, R. (1993) "Experimental Results on Interactive Competitive Guessing." Discussion Paper No. B-236, University of Bonn.

[30] Potters, J. C., De Vries, C. G., and Van Winden, F. (1998) "An experimental examination of rational rent seeking". European Journal of Political Economy, $14,783-800$.

[31] Parco, J., Rapoport, A., and Amaldoss, W. (2005) "Two-stage contests with budget constraints: An experimental study". Journal of Mathematical Psychology, 49, 320-338.

[32] Selten, R. (1970). Preispolitik der Mehrproduktenunternehmung in der Statischen Theorie (First ed.). Springer Verlag, Berlin.

[33] Sheremeta, R. M., and Zhang, J. (2010). "Can groups solve the problem of over-bidding in contests?", Social Choice and Welfare, 35, 175-197.

[34] Sheremeta, R. M. (2011). "Contest design: An experimental investigation". Economic Inquiry, 49, 573-590.

[35] Sheremeta, R. M. (2013). "Overbidding and Heterogenous Behavior in Contest Experiments". Journal of Economic Surveys, 27 (3), 491-514.

[36] Stahl. D., and P. Wilson (1994) "Experimental Evidence on Players' Models of Other Players," Journal of Economic Behavior and Organization, 25, 309-327.

[37] Stahl. D., and P. Wilson (1995) "On Players' Models of Other Players: Theory and Experimental Evidence," Games and Economic Behavior.

[38] Tullock, G. (1980) "Efficient Rent Seeking", in J. M. Buchanan, R. D. Tollison, \& G. Tullock(Eds.), Toward a theory of the rent-seeking society. Texas A\&M University Press. 